\title{
Degrading river network due to urbanization in Yangtze River Delta
}

\author{
HAN Longfei ${ }^{1},{ }^{*} X U$ Youpeng ${ }^{1}$, LEI Chaogui ${ }^{1}$, YANG Liu ${ }^{1}$, DENG Xiaojun ${ }^{1}$, \\ HU Chunsheng ${ }^{1,2}$, XU Guanglai ${ }^{2}$ \\ 1. School of Geographic and Oceanographic Sciences, Nanjing University, Nanjing 210023, China; \\ 2. College of Territorial Resources and Tourism, Anhui Normal University, Wuhu 241000, Anhui, China
}

\begin{abstract}
Evolution of river systems under the background of human activities has been a heated topic among geographers and hydrologists. Spatial and temporal variations of river systems during the 1960s-2010s in the Yangtze River Delta (YRD) were investigated based on streams derived from the topographic maps in the 1960s, 1980s and 2010s. A list of indices, drainage density $(D d)$, water surface ratio $(W S R)$, ratio of area to length of main streams $(R)$, evolution coefficient of tributaries $(K)$ and box dimension $(D)$, were classified into three types (quantitative, structural, and complex indices) and used to quantify the variations of stream structure. Results showed that: (1) quantitative indices ( $D d, W S R)$ presented decreasing trend in the past 50 years, and $D d$ in Wuchengxiyu, Hangjiahu and Yindongnan have decreased most, about 20\%. Structurally, the Qinhuai River basin was characterized by significant upward $R$, and $K$ value in Hangjiahu went down dramatically by $46.8 \%$ during the 1960s-2010s. Decreasing tendency in $D$ was found dominating across the YRD, and decreasing magnitude in Wuchengxiyu and Hangjiahu peaks for $7.8 \%$ and $6.5 \%$, respectively in the YRD. (2) Urbanization affected the spatial pattern of river system, and areas with high level of urbanization exhibited least $D d\left(2.18 \mathrm{~km} / \mathrm{km}^{2}\right), W S R(6.52 \%), K(2.64)$ and $D(1.42)$, compared to moderate and low levels of urbanization. (3) Urbanization also affected the evolution of stream system. In the past 50 years, areas with high level of urbanization showed compelling decreasing tendency in quantitative $(27.2 \%$ and $19.3 \%)$ and complex indices $(4.9 \%)$ and trend of enlarging of main rivers $(4.5 \%$ and $7.9 \%$ in periods of the $1960 \mathrm{~s}-1980 \mathrm{~s}$ and the 1980s-2010s). In the recent 30 years, areas with low level of urbanization were detected with significant downward trend in $D d$ and $K$. (4) Expanding of urban land, construction of hydraulic engineering and irrigation and water conservancy activities were the main means which degraded the river system in the YRD.
\end{abstract}

Keywords: stream structure; spatial and temporal change; urbanization; Yangtze River Delta

Received: 2015-11-12 Accepted: 2015-12-15

Foundation: National Natural Science Foundation of China, No.41371046; The Commonwealth and Specialized Program for Scientific Research, Ministry of Water Resources of China, No.201201072, No.201301075; Natural Science Foundation of Jiangsu Province, No.BK20131278

Author: Han Longfei (1988-), PhD Candidate, specialized in urban hydrology. E-mail: hanlf.nju@gmail.com

*Corresponding author: Xu Youpeng, Professor, E-mail: xuyp305@163.com 


\section{Introduction}

River network is one of the most important ecosystems on earth (Ni and Liu, 2006), and carrier for the formation and evolution of water resource, supporting the development of human society essentially (Li et al., 2011). River evolution in East China is influenced by a combination of natural factors and human activities in the long history, but streams have been marked by strong human interference in recent 50 years. Both quantity and morphology were altered by the human activities, especially urbanization which was denoted by highly-intense, large-covering and long-lasting alteration. Then, the function of regulating and storing pollutants that rivers should have has changed, causing the increasing flood hazards and serious water environmental issues which put restrictions on the sustainability of the human society $(\mathrm{Xu}, 2012)$.

As early as in 1865, Marsh (1865) had found that human activities could change the evolution of rivers. Horton (1945) proposed a stream order system and found the two famous stream laws - the laws of stream numbers and length. The stream order system was then revised by Strahler (1957) and widely used in the research of stream structure. The study on the relationship between urbanization and rivers started in the 1960s, and early researches showed enlarged channel width and cross-section (Gregory et al., 1992; Hammer, 1972) and increased drainage density (Graf, 1977) in the urbanized areas. Vanacker et al.'s research (2005) on the Andes Mountains showed that stream channels narrowed by over $45 \%$ and riverbed deepened by over 1 meter as a result of LUCC (land use and cover change). But the process known as headstream burial where streams are paved over and altered into roads, buildings or other impervious areas is probably the most extreme impact of urbanization on streams. Recent investigations have shown that stream burial is occurring at high rate all around the world. In Baltimore City, USA, 66\% of the streams have been buried (Elmore and Kaushal, 2008) and drainage density in the Rock Creek watershed in Maryland has been reduced by $58 \%$ due to urbanization (Meyer et al., 2001). The headwater streams can provide the urbanizing watershed with an ability to hold and store water, and effectively retain the sediments and transform and retain nutrients and contaminations, thereby providing water-quality benefits downstream (Dunne and Leopold, 1978). Therefore, disappearing headwater streams not only increases risk of vulnerability to flood, but also affects the fluvial ecosystem on a great deal.

China has stepped into the period of high-speed urbanization since the 1980s, thus, investigations of impact of urbanization on rivers lag behind those in the world. Past researches in China were mainly done in highly developed area, such as the Yangtze River Delta (Chen et al., 2002; Han et al., 2013; Han et al., 2015; Ji et al., 2014; Xu et al., 2013; Yang et al., 2004; Yuan et al., 2005; Yuan et al., 2007) and the Pearl River Delta (Huang et al., 2008; Zhou et al., 2008). Han and Mao (1997) concluded that channels around Taihu Lake had declined from over 300 in the early 20th century to 125 in 1993. Chen et al. (2002) analyzed the river network evolution in the process of urbanization in Shanghai, and results showed that stream numbers decreased a lot, thus influencing the natural drainage ability of rivers. Yang et al. (2004) revealed that urbanization was an important factor altering the structure of tidal river system. Zhou et al. (2006) showed in the investigation on the Beijing-Tianjin reach of the Yongding River that stream length and number in these areas had decreased by $20.5 \%$ and $36.4 \%$ in recent 40 years, respectively, and stream structure was simplified. Besides, urbanization in China has been running deep in recent years, and discrepancy of tradeoff be- 
tween city expanding and river network has become increasingly salient, in the wake of which, a serial of flood and fluvial environmental issues arrived. Therefore, impacts that urbanization has on the river networks have currently drawn more attention among government, scholars and the public.

Yangtze River Delta is one of the most developed and densely populated areas in China. Fierce human activities dramatically have damaged the river network system in the past 30 years, inducing decreasing stream density and water surface. There have been some investigations of stream response to human activities, and they were mainly restricted to regional case study, generally taking a city as an example. However, there is a lack of comparative investigation on the whole Yangtze River Delta. Therefore, this paper choose five water conversation districts, Yangchengdianmao (YCDM), Wuchengxiyu (WCXY), Hangjiahu (HJH), Qinhuai River (QHH) and Yongcaopu (YCP), as the study area since they cover most of the developed areas in the Yangtze River Delta. Based on extracted river network of three periods (1960s, 1980s, and 2010s), indices system of river geomorphology were established and used for analysis on the temporal and spatial characteristics of the river network across the Yangtze River Delta in the past 50 years. By investigating the variation of river network of areas with different degrees of urbanization, we try to seek the process of evolution of stream networks influenced by the urbanization.

\section{Methods}

\subsection{Study area}

The Yangtze River Delta (YRD), located in East China $\left(29^{\circ} 12^{\prime} \mathrm{N}-33^{\circ} 19^{\prime} \mathrm{N}, 118^{\circ} 19^{\prime} \mathrm{E}-\right.$ $122^{\circ} 19^{\prime} \mathrm{E}$, Figure 1), covers an area of $9.54 \times 10^{4} \mathrm{~km}^{2}$ (Figure 1). The region is mostly controlled by the typical subtropical East Asian monsoon with four distinct seasons which is characterized by mild and wet climate. The average annual precipitation is over $1000 \mathrm{~mm}$, ranging from 729.7 to $1526.2 \mathrm{~mm}$. The precipitation is mainly concentrated in May to September, dominated by the pulm rains and typhoon. The YRD is featured by alluvial plains and hills, $85.3 \%$ of which is below $200 \mathrm{~m}$ with an average elevation of $83.3 \mathrm{~m}$, so this area is prone to flood. Mountains and hills are mainly located in the south and southeast of the YRD with the highest elevation of $1635 \mathrm{~m}$ while alluvial plains are mainly located in the north and east of the YRD with mean elevation lower than $50 \mathrm{~m}$. Large rivers run across the YRD, e.g., the Huaihe River, Yangtze River and Qiantang River from north to south, respectively. River networks develop well and are densely distributed in this region, with a drainage density of over $3.0 \mathrm{~km} / \mathrm{km}^{2}$, and there are also many lakes in the YRD, the largest one is Taihu Lake with an area of $2427.8 \mathrm{~km}^{2}$.

The YRD is divided into 17 water conservation districts based on stream channels variation, topographical elevation deviations and flood characteristics. The Taihu Lake basin is the largest watershed over the YRD, and its catchment area is $36,900 \mathrm{~km}^{2}$, accounting for $38.7 \%$ of the YRD. River networks in this basin are divided into two parts by the lake: upstream network and downstream network. Downstream part is characterized by plain river network, including Huangpu river system in the east (where Shanghai is located), streams networks along the Yangtze River in the north and Hangzhou Bay river system in the south. There are 7 water conservation districts in the Taihu basin, and the selected ones in this paper are among the downstream part, i.e. Wuchengxiyu, Yangchengdianmao and Hangjiahu. 
Qinhuai River basin is mainly located in the city of Nanjing, and its main stream flows directly into the Yangtze River at Sanchakou. Streams run crisscross in the middle and lower reaches of the basin, typical of plain river network. Yindongnan (YDN) plain of YCP river basin, located in coastal areas southeast of the $\mathrm{YRD}$, is also featured by plain river network.

We select five water conservation districts, Wangchengxiyu (A), Yangchendianmao (B), Hangjiahu (C), Qinhuai River basin (D) and Yindongnan (E). The boundary line between regions A and B is Wangyu River, one of most important discharging conduits in the Taihu basin. Taipu River, another one, separates regions B and C, and Qiantang River divides regions $\mathrm{C}$ and $\mathrm{D}$. Wuchengxiyu (region $\mathrm{A}$ ) leads the stream density, and region $\mathrm{B}$ shares the highest water surface ratio. Previous work in the YRD megalopolitan region using remote sensing techniques has shown a $75.8 \%$ increase (about $1.37 \times 10^{5}$ $\mathrm{hm}^{2}$ ) in urban area from 19902005 (Tian et al., 2011), with the highest urban expansion intensity from 2000-2010 (Xiao et al., 2014). The districts selected for this study includes 8 cities, i.e. Suzhou, Wuxi, Changzhou, Hangzhou, Jiaxing, Huzhou, Nanjing and Ningbo, which are among the most developed areas across the YRD. River networks system in the YRD weakened a lot during the past half century, and are, therefore, likely to be typical of urbanizing regions in the developing countries worldwide.

\subsection{Data and processing}

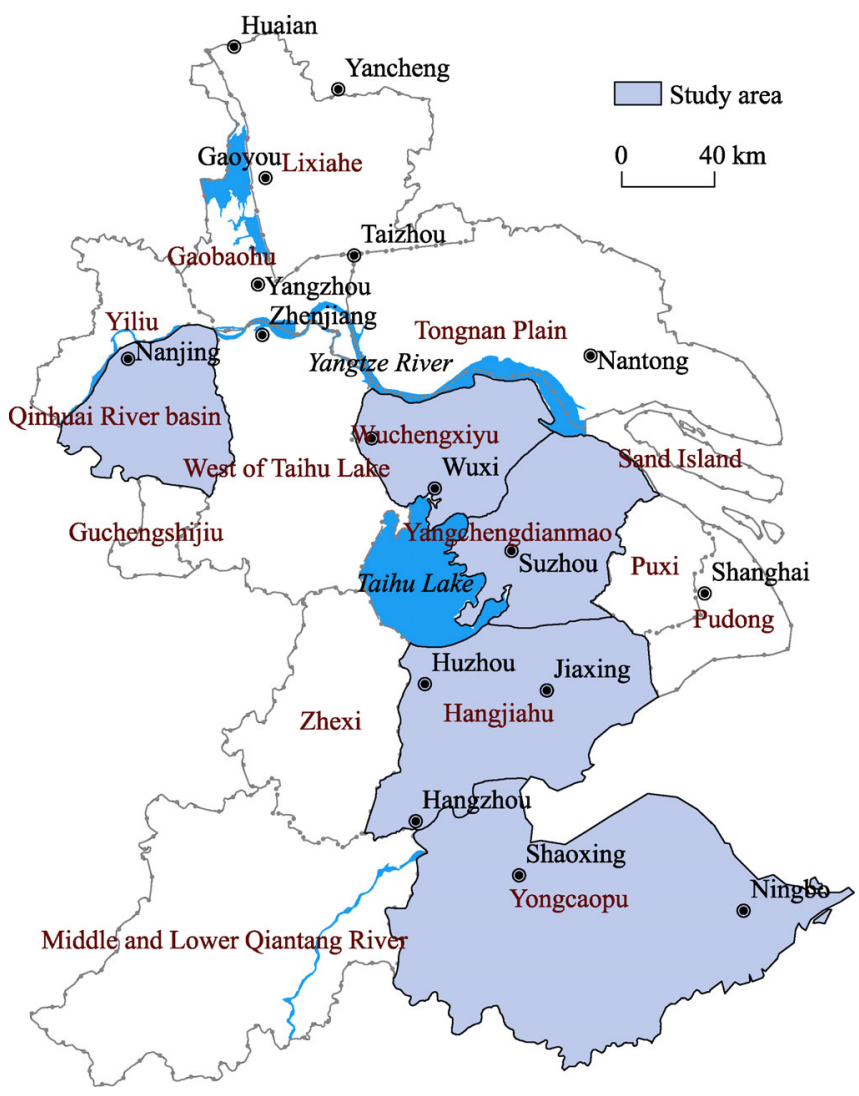

Figure 1 Location of the Yangtze River Delta

River networks were extracted using three periods of topographic maps in the 1960s, 1980s and 2010s. The topographic maps display all streams in four regions (A, B, C and D) at a scale of 1:50,000. These were first produced by the Chinese military administration of surveying and mapping in the $1960 \mathrm{~s}$, with the final version released by the national administration of surveying, mapping and geoinformation in 2009. The ones in the 1960s and 1980s were in the paper form, and all the streams were delineated manually in ArcMap 9.3. The final version in 2009 was GIS layer displaying all the streams, and these streams were updated and checked by a combination of field and space photography ( $2 \mathrm{~m}$ resolution) provided by the Google Map in 2014. Steam data in region E were based on the digital topographic maps at a scale of 1:10,000 in 1999, 2003 and 2010. Having considering Strahler's (1957) stream order system and complexity of plain river network, the streams orders were 
so chosen that large streams with a width over $20 \mathrm{~m}$ are of the 1 st order and defined as main streams; tributaries are those with channel width below $20 \mathrm{~m}$, and streams with a width between 10 and $20 \mathrm{~m}$ are of the 2 nd order; 3rd order streams are those with a width less than $10 \mathrm{~m}$. Main steams are important conduits of flood discharging, while tributaries play key roles in regulating and storing water.

Extracting streams in the paper form includes scanning of the topographic maps and georeferencing in ArcGis 9.3. According to the stream order system described above, we delineated the streams of the three orders of the 1960s and 1980s, and obtained two-period stream through mosaic and clip operation and topology test of delineated lines. The GIS layer streams in the 2010s were extracted directly in ArcGis without manual delineation. Then, we calculated the indices of streams of the three periods in ArcGis. Additionally, Landsat images (30-m resolution) in 2010 provided by US Geological Survey (USGS) were used, and decision-tree classification was applied to the interpretation of urban land in Envi4.7 to acquire the urbanization degree in selected cities.

\subsection{Indices of stream structure}

Five indices were selected in this paper to describe the geomorphic characteristics of the river networks, including drainage density $(D d)$, water surface ratio $(W S R)$, development coefficient of tributaries $(K)$, ratio of area to length of main streams $(R)$ and box dimension $(D)$, as listed in Table 1.

Table 1 Definitions of stream structure indices in this study

\begin{tabular}{|c|c|c|c|c|}
\hline Indices & Definitions & Formulas & Units & Explanations \\
\hline$D d$ & $\begin{array}{l}\text { Drainage } \\
\text { density }\end{array}$ & $\begin{array}{l}D d=L / A, L \text { is total stream length in the } \\
\text { basin, } A \text { is area of the basin }\end{array}$ & $\mathrm{km} / \mathrm{km}^{2}$ & $\begin{array}{l}D d \text { was firstly introduced } \\
\text { by Horton (1945) to indi- } \\
\text { cate the drainage ability }\end{array}$ \\
\hline$W S R$ & $\begin{array}{l}\text { Water surface } \\
\text { ratio }\end{array}$ & $\begin{array}{l}W S R=\left(A_{\mathrm{w}} / A\right) \times 100 \%, A_{\mathrm{w}} \text { is total water area, } \\
\text { including the water area of streams, ponds } \\
\text { and lakes }\end{array}$ & $\%$ & $\begin{array}{l}W S R \text { denotes the regulat- } \\
\text { ing and storing ability }\end{array}$ \\
\hline$K$ & $\begin{array}{l}\text { Development } \\
\text { coefficient of } \\
\text { tributaries }\end{array}$ & $\begin{array}{l}K=L_{\mathrm{b}} / L_{\mathrm{m}}, L_{\mathrm{b}} \text { is length of tributaries, } L_{\mathrm{m}} \text { is } \\
\text { length of main streams }\end{array}$ & $\begin{array}{l}\text { No } \\
\text { dimension }\end{array}$ & $\begin{array}{l}K \text { refers to developing } \\
\text { degree of tributaries }\end{array}$ \\
\hline$R$ & $\begin{array}{l}\text { Ratio of area } \\
\text { to length of } \\
\text { main streams }\end{array}$ & $\begin{array}{l}R=A_{\mathrm{m}} / L_{\mathrm{m}}, A_{\mathrm{m}} \text { is area of the main streams, } \\
L_{\mathrm{m}} \text { is length of main streams }\end{array}$ & $\mathrm{km}^{2} / \mathrm{km}$ & $\begin{array}{l}R \text { is the average width of } \\
\text { main streams, indicating } \\
\text { developing degree of } \\
\text { main stream }\end{array}$ \\
\hline$D$ & $\begin{array}{l}\text { Box dimen- } \\
\text { sion }\end{array}$ & $\begin{array}{l}\text { Intersecting the river networks with square } \\
\text { boxes with side length r, count the number } \\
N(r) \text { of boxes with stream segments. } N(r) \\
\text { increases with decreasing r of the box, and } \\
\text { we can get a serial of } r-N(r) \text {. }\end{array}$ & $\begin{array}{l}\text { No } \\
\text { dimension }\end{array}$ & $\begin{array}{l}\text { Fractal theory was first } \\
\text { introduced to geography, } \\
\text { and it denotes the com- } \\
\text { plexity of the pattern of } \\
\text { river network (Zhang et } \\
\text { al., 2015). }\end{array}$ \\
\hline
\end{tabular}

\section{Results}

\subsection{Component characteristic of river system}

Having calculated the number and length of streams of 3 orders, we get the structure of the river network as shown in Figure 2. Both number and length of main streams contribute 
small proportion to whole river network, and tributaries contribute large proportion, especially stream of 3 rd order, contributing the most, which is typical in region A and B. But the difference of contribution between main streams and tributaries is not quite much in region $\mathrm{C}$ and $\mathrm{D}$, indicating that development degrees of tributaries in these two areas are not as high as abdomen part of Taihu basin (region A and B). In region E, stream length of 1st and 3rd orders is not in accordance with stream number, and average length of 1st order stream (4.63 $\mathrm{km}$ ) is far higher than $3 \mathrm{rd}$ order indicating that tributaries are highly fragmented in this area.
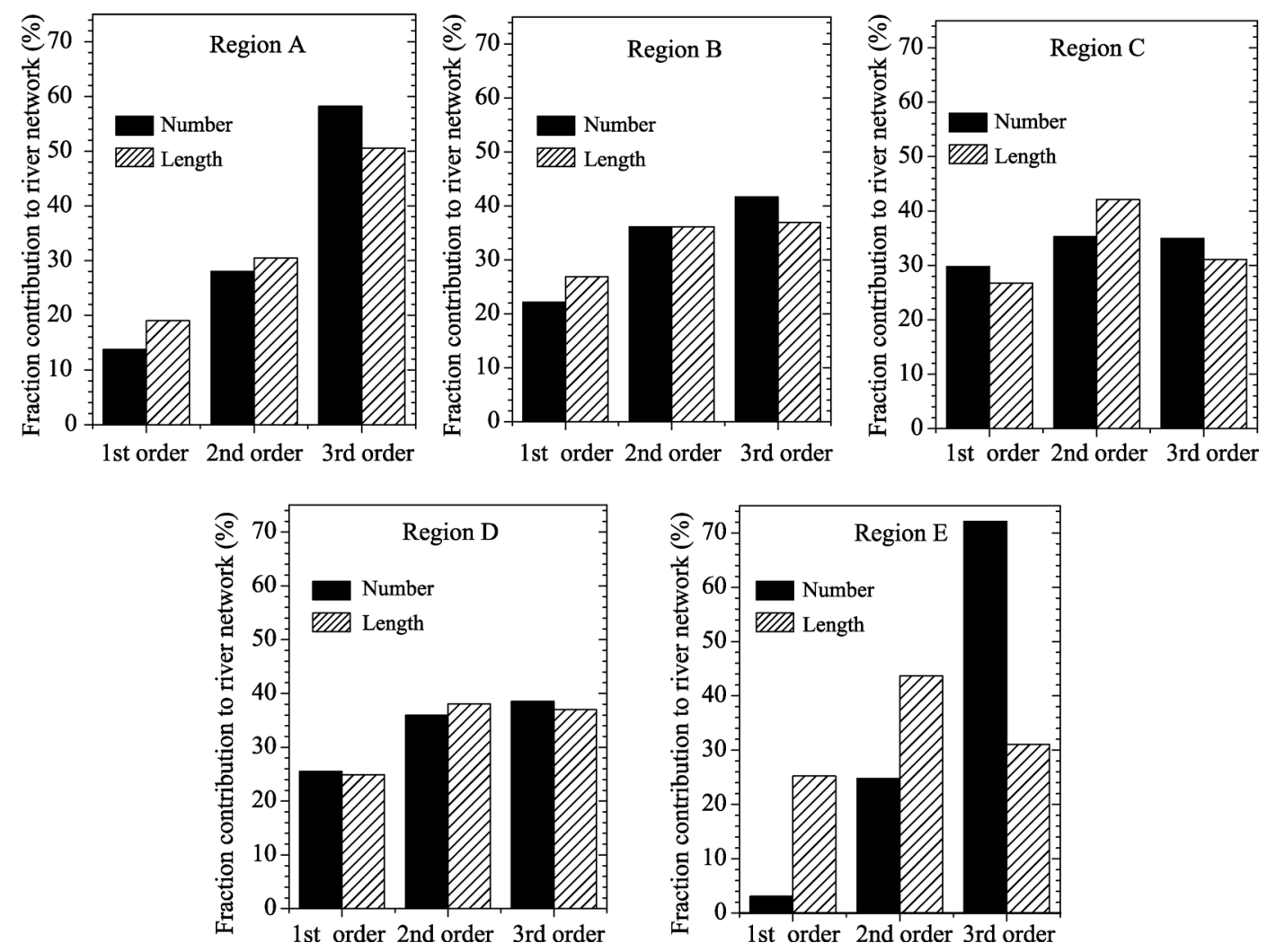

Figure 2 Fraction contributions of different streams orders to river network in number and length

\subsection{Variation of quantity of river system}

Drainage density $(D d)$ and water surface ratio $(W S R)$ are used to describe the quantitative characteristic of river network. $D d$ in regions A, B and C, from Figure 3, were the highest in the study area and reached more than $3.5 \mathrm{~km} / \mathrm{km}^{2}$ in $D d$. $D d$ in Shanghai approximated this value in 2000, as shown by Yang et al. (2004). YDN (region E) followed these regions in Dd, while QHH (region D) shared the lowest $D d$, only 1.1 in the 2010s. The differences among these areas is contributed by the topology of them that region A, B, C and Shanghai are located in the lower Taihu basin, while region D is featured by hills. River network has high degree of development in the lower Taihu basin which is characterized by plain and many human-made rivers were constructed in the historic period for irrigation and transportation. But it is quite low in the degree of river development in hills. Compared with the YRD, the 
Pearl River Delta, second largest delta in China, has far lower Dd (Table 2) which is caused by scale of the extracted topographic maps. Extraction of steams in the Pearl River Delta is based on the maps of 1:100,000 scale, while it is 1:50,000 in my research on the YRD. Overall, $D d$ in the YRD exhibited a decreasing tendency during the 1960s-2010s that regions A, C and E decreased significantly (about 20\%) while decreasing magnitude of regions $\mathrm{B}$ and $\mathrm{D}$ were much smaller, $3.8 \%$ and $9.1 \%$, respectively.
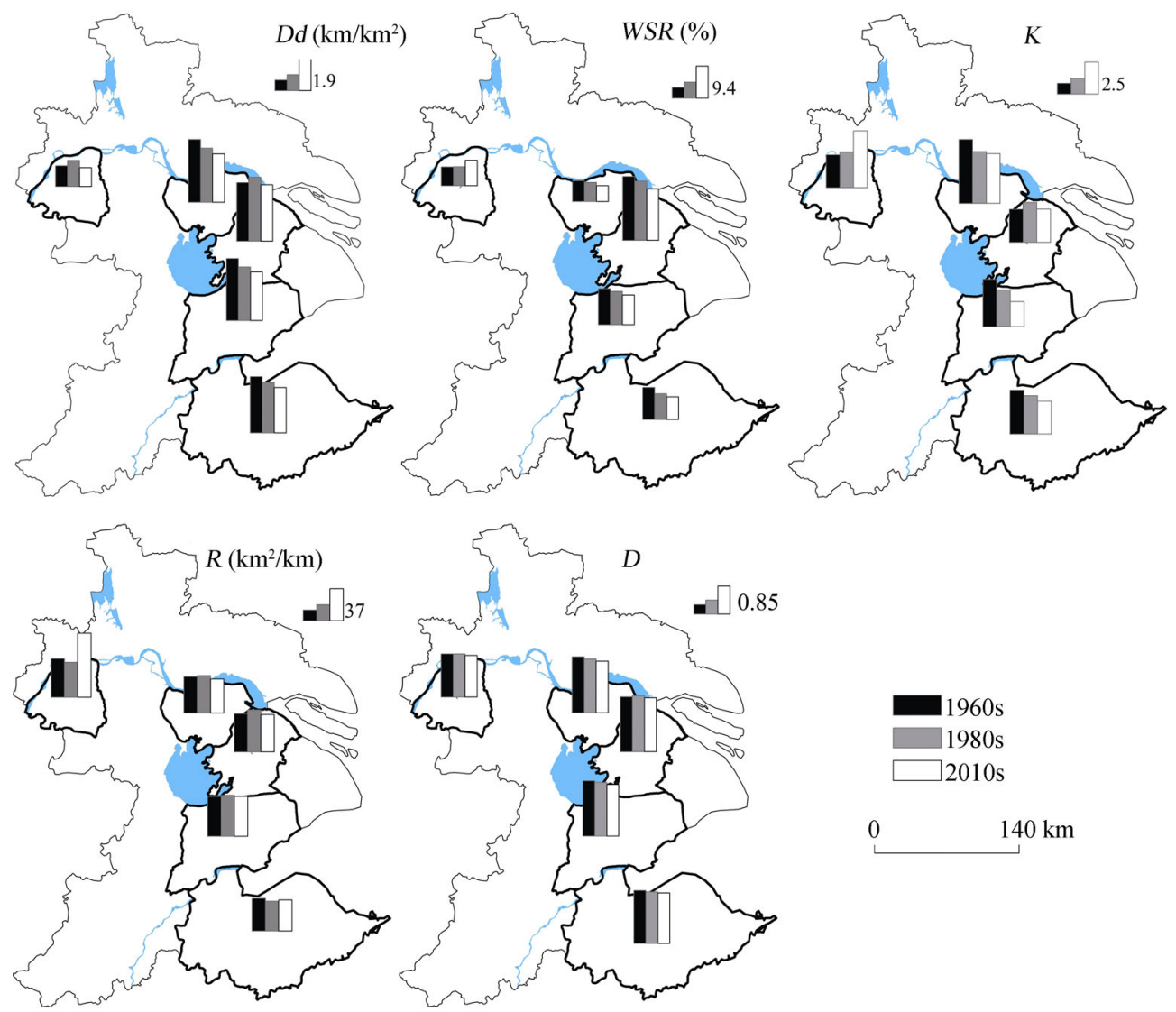

Figure 3 Characteristics of stream structure in the Yangtze River Delta in the past 50 years

YCDM (region B) enjoyed the highest WSR for its densely-dotted lakes, $18.9 \%$ in the 1960s (Figure 3 and Table 2), then followed by region $C(8.6 \%-10.6 \%)$ for its adjacency to region $\mathrm{B}$ in the north. But WSR in regions $\mathrm{A}, \mathrm{B}$ and Shanghai was low, about $5.0 \%-7.5 \%$. All the regions except $\mathrm{QHH}$ showed a large downward magnitude in WSR. Decreasing magnitude in WSR peaked for region $\mathrm{E}(29.5 \%)$, followed by regions $\mathrm{A}, \mathrm{B}$ and $\mathrm{C}$, being $23.5 \%$, $19.4 \%$ and $17.5 \%$, respectively (Table 2). During the $1960 \mathrm{~s}-1980 \mathrm{~s}$, the decreasing magnitude for $\mathrm{A}, \mathrm{B}$ and $\mathrm{C}$ is $8.3 \%, 7.4 \%$ and $7.1 \%$, respectively, while they were $16.55 \%, 13.05 \%$ and $11.3 \%$, respectively, during the $1980 \mathrm{~s}-2010 \mathrm{~s}$, indicating a intense downward trend. But WSR in QHH increased in the past 50 years, and it was quite obvious during the $1980 \mathrm{~s}-2010 \mathrm{~s}$ (34.2\%), which is caused by the greatly increasing ponds (Han et al., 2013; Ji et al., 2014). 
Table 2 Change of streams structure in different regions in the past 50 years

\begin{tabular}{|c|c|c|c|c|c|c|c|c|}
\hline \multirow[b]{2}{*}{ Indices } & \multirow[b]{2}{*}{ Period } & \multicolumn{5}{|c|}{ Study area } & \multicolumn{2}{|c|}{ Other areas } \\
\hline & & A & B & $\mathrm{C}$ & $\mathrm{D}$ & $\mathrm{E}^{*}$ & $\begin{array}{c}\text { Shanghai* } \\
\text { (Yang et al., 2004; } \\
\text { Yuan et al., 2005) }\end{array}$ & $\begin{array}{l}\text { Pearl River } \\
\text { Delta* (Zhou } \\
\text { et al., 2008) }\end{array}$ \\
\hline \multirow[t]{2}{*}{ Area $\left(\mathrm{km}^{2}\right)$} & - & 3841.0 & 4914.0 & 7621.0 & 497.1 & 476.1 & 4962.5 & 1991.8 \\
\hline & $1960 \mathrm{~s}$ & 3.80 & 3.54 & 3.75 & 1.25 & 3.40 & & 0.86 \\
\hline \multirow{3}{*}{$D d$} & $1980 \mathrm{~s}$ & 3.27 & 3.87 & 3.24 & 1.58 & 3.06 & 3.45 & 0.84 \\
\hline & $2010 \mathrm{~s}$ & 2.93 & 3.41 & 2.93 & 1.14 & 2.75 & & 0.65 \\
\hline & $1960 \mathrm{~s}$ & 6.10 & 18.86 & 10.63 & 5.53 & 9.50 & & \\
\hline \multirow[t]{3}{*}{$W S R$} & $1980 \mathrm{~s}$ & 5.59 & 17.47 & 9.88 & 5.60 & 7.60 & 5.62 & \\
\hline & $2010 \mathrm{~s}$ & 4.66 & 15.20 & 8.76 & 7.52 & 6.70 & & \\
\hline & $1960 \mathrm{~s}$ & 4.93 & 2.54 & 3.65 & 2.52 & 3.39 & & 4.42 \\
\hline \multirow[t]{3}{*}{$K$} & $1980 \mathrm{~s}$ & 4.00 & 3.07 & 2.83 & 2.74 & 2.96 & 3.39 & 3.04 \\
\hline & $2010 \mathrm{~s}$ & 3.83 & 2.56 & 1.94 & 4.37 & 2.53 & & 3.50 \\
\hline & $1960 \mathrm{~s}$ & 41.53 & 43.75 & 45.54 & 44.11 & 37.40 & & \\
\hline \multirow[t]{3}{*}{$R$} & $1980 \mathrm{~s}$ & 42.83 & 48.22 & 47.03 & 39.96 & 34.10 & & \\
\hline & $2010 \mathrm{~s}$ & 38.85 & 42.94 & 45.66 & 73.23 & 35.60 & & \\
\hline & $1960 \mathrm{~s}$ & 1.71 & 1.68 & 1.69 & 1.32 & 1.62 & & \\
\hline \multirow[t]{2}{*}{$D$} & $1980 \mathrm{~s}$ & 1.65 & 1.71 & 1.64 & 1.32 & 1.58 & 1.40 & \\
\hline & $2010 \mathrm{~s}$ & 1.58 & 1.65 & 1.58 & 1.27 & 1.54 & & \\
\hline
\end{tabular}

Note: *The three periods of region D is 1990, 2003 and 2010, respectively, and there is merely one period (2010) in Shanghai, three periods (1980, 1985 and 2005) in Pearl River Delta; the tributaries development coefficient in Shanghai and Pearl River Delta were calculated with the data provided in their papers; Calculation of WSR in Shanghai does not include lake areas, and its fractal value is computed based on Horton law.

\subsection{Variation of structure of river network}

Ratio of area to length of main stream $(R)$ and development coefficient of tributaries $(K)$ are applied to describe stream structure. $R$ values, i.e. average width of main stream, did not vary that much in the subdistricts of the study area (Figure 3). Main streams have the priority of protection for its key function of discharging, and construction of new main streams and human-made dredging and widening of existing large rivers are steps that department of water resources has taken to alleviate the severe situation of flood caused by fast urbanization. Obvious change was detected in Qinhuai River basin that $R$ value increased from 40.0 in the 1980 s to 73.2 in the 2010s. This is due to the newly-built New Qinhuai River, one of two most important conduit of discharging flood, and its mean width is as high as $112 \mathrm{~m}$. But variation of $R$ value in other regions in the past 50 years was not large, with regions $\mathrm{A}$, $\mathrm{B}$ and $\mathrm{E}$ decreasing slightly and region $\mathrm{C}$ increasing a little.

But $K$ values were quite different among the study regions (Figure 3 and Table 2). Region A shared the highest $K$, averaging 4.3 in the three periods, as seen in Table 2, while regions $\mathrm{B}$ and D exhibited relatively low values. It was 3.39 in Shanghai, and averaged 3.65 from 1980 to 2005 in the Pearl River Delta. In the past 50 years, there has seen decreasing $K$ in regions $\mathrm{A}, \mathrm{C}$ and $\mathrm{E}$, especially in $\mathrm{C}$, in which $K$ value decreased by $46.8 \%$ during the 1960s-2010s, facing serious problems in stability of river structure. However, it is surprising 
that $K$ in the Qinhuai River basin increased by $73.0 \%$, and it is, further investigation shows, due to great decrease of the main stream's length. In the 1960s, the main stream's length comprised $28.4 \%$ of the total, but in the 2010 s, this figure dropped to $18.6 \%$. Considering downward trend of both main streams and tributaries, the fact that decreasing magnitude of the main streams was higher than the tributaries accounted for the increasing trend of $K$. In region B, $K$ did not have fluctuations in recent 50 years.

\subsection{Variation of river network complexity}

Fractal dimension $(D)$ was positively related to drainage density $(D d)$, and it had apparently varying spatial distribution. The lower Taihu basin exhibited the largest $D d$ as well as $D$, while QHH shared the lowest $D$ with the smallest $D d$. In the past 50 years, all the regions showed decreasing tendency of $D$, with values of $1.32-1.71$ in the 1960 s and $1980 \mathrm{~s}$ and $1.27-1.65$ in the $2010 \mathrm{~s}$. Among these, regions $\mathrm{A}$ and $\mathrm{C}$ featured by dense $D d$ showed larger decreasing magnitude $(7.8 \%$ and $6.5 \%$ ), while it was just $2.0 \%$ in region B characterized by densely-dotted lakes.

\section{Discussions}

\subsection{Influence of urbanization on river network}

Stream structure has being changing as disturbed by intense human activities. Variation of the stream structure in the YRD, one of the most developed areas in China, is mainly caused by the human intervention. In order to investigate the impact of urbanization on river structure, we classified administrative divisions in the YRD into three types based on the urbanization degree, high, medium and low levels of urbanization (Table 3).

Urbanization is characterized by increasing imperviousness in short time, thus accountable for the decreasing quantity of streams. In Shanghai, China, highly urbanized area shared the lowest WSR (Yuan et al., 2005). In Baltimore, USA, drainage density in urban area decreased by $66 \%$ (Elmore and Kaushal, 2008), the value dropped to $18 \%$ in suburbs. But the variation of $D d$ in the city of Shenzhen, Pearl River Delta, was not significantly related to the degree of urbanization spatially (Huang et al., 2008). In our study, areas with high level of urbanization shared the lowest $D d$, averaging $2.18 \mathrm{~km} / \mathrm{km}^{2}$ in three periods (Table 4), while it is far higher in medium and low levels of urbanization, averaging 3.83 and 3.63, respectively. During the 1960s-1980s, $D d$ showed a decreasing tendency except in medium urbanization degree. Increasing $D d$ in this area was caused by agricultural activities during this period which was confirmed by in situ investigation in the year of 2014. Channels and canals were constructed in the 1980 s to meet the increasing need for irrigation during agricultural activities, and they were identified as streams in the extract of river networks in this study. So $D d$ during this period in Kunshan, Taicang, Changshu, Wuxian and Wujiang has increased by $4.6 \%, 13.1 \%, 11.5 \%, 12.1 \%$ and $8.0 \%$, respectively, which is not shown in Table 4 . However, during the 1980s-2010s all the areas showed decreasing trend, and high level of urbanization led in the decreasing magnitude $(23.1 \%)$, followed by medium and low levels, $10.0 \%$ and $14.6 \%$, respectively, which is consistent with the result in Baltimore City(Elmore and Kaushal, 2008). 
Table 3 Administrative divisions with different levels of urbanization in the Yangtze River Delta

\begin{tabular}{lll}
\hline & \multicolumn{1}{c}{ Administrative division } & Urbanization degree (\%)* \\
\hline High level of urbanization & Municipal district of Changzhou & 58.15 \\
& Municipal district of Suzhou & 57.76 \\
& Municipal district of Wuxi & 49.94 \\
& Municipal district of Hangzhou & 46.79 \\
& Kunshan County & 39.68 \\
Medium level of urbanization & Zhangjiagang County & 38.38 \\
& Taicang County & 35.62 \\
& Changsu County & 34.64 \\
& Wuxian County & 31.93 \\
Low level of urbanization & Wujiang County & 30.18 \\
\hline
\end{tabular}

Note: *Urbanization level is calculated by dividing construction area by its total area, and construction area was acquired by remote sensing interpretation.

Similar spatial distribution was found for the distribution of WSR to Dd (Table 4). Areas with high level urbanization exhibited the lowest $W S R$, averaging $6.5 \%$, followed by $13.35 \%$ of medium level urbanization area, and WSR in low level of urbanization was maximum, which was similar to conclusions in Shanghai. During the 1960s-2010s, WSR in the both highly and moderately urbanized areas decreased by approximately $20 \%$, while it was $11.20 \%$ in lowly urbanized area.

Two ways of human activities, headwater burial and dredging of main streams alter the characteristic of stream structure (Xu et al., 2013). Inferred from Table 4, $K$ value in highly urbanized area is the smallest, averaging 2.64 in the three periods, followed by 4.71 of moderately urbanized area, and values peak (6.07) for lowly urbanized area. In the past 50 years, tributaries decreased a great deal in high and low degree of urbanization, with decreasing rate of $28.45 \%$ and $68.34 \%$, respectively, while the downward magnitude in medium degree of urbanization is rather low. Moreover, the downward tendency of $K$ in these two areas have intensified in the past 50 years, since the decreasing rates during the $1960 \mathrm{~s}-1980$ s were $1.27 \%$ and $32.15 \%$, respectively, and increased to $27.53 \%$ and $53.33 \%$ during the 1980s-2010s. It is noted that headwater in lowly urbanized areas were also faced with severe disappearing as urbanization deepened in recent 30 years, for example, $K$ value in this area was just 2.89 in the 2010s, a little higher than in highly urbanized area.

Most dominant impact of urbanization on main streams can be detected in highly urbanized area. $R$ value peaks in this area, averaging 43.27 in the three periods, followed by 43.16 in medium level of urbanization, and low urbanized area shared the lowest $R$ value. And widening tendency of main streams has intensified in highly urbanized area since $R$ value had climbed from 40.91 in the 1960 s to 42.76 in the 1980 s by $4.53 \%$ and increasing rate during the $1980 \mathrm{~s}-2010 \mathrm{~s}$ rose to $7.89 \%$. But this tendency was not apparent in medium and low degrees of urbanization, and changing rates in these two areas were $2.36 \%$ and $-0.97 \%$, respectively. 
Table 4 Characteristics of stream structure in administrative divisions with different levels of urbanization in the Yangtze River Delta

\begin{tabular}{|c|c|c|c|c|}
\hline \multirow{2}{*}{ Indices } & \multirow{2}{*}{ Periods } & \multicolumn{3}{|c|}{ Areas of different urbanization degree } \\
\hline & & High level & Medium level & Low level \\
\hline \multirow{3}{*}{$D d$} & $1960 \mathrm{~s}$ & 2.45 & 3.80 & 4.47 \\
\hline & $1980 \mathrm{~s}$ & 2.32 & 4.04 & 3.46 \\
\hline & $2010 \mathrm{~s}$ & 1.78 & 3.64 & 2.96 \\
\hline \multirow{3}{*}{$W S R$} & $1960 \mathrm{~s}$ & 6.88 & 14.69 & 17.23 \\
\hline & $1980 \mathrm{~s}$ & 7.14 & 13.51 & 16.25 \\
\hline & $2010 \mathrm{~s}$ & 5.55 & 11.85 & 15.30 \\
\hline \multirow{3}{*}{ K } & $1960 \mathrm{~s}$ & 2.93 & 4.90 & 9.12 \\
\hline & $1980 \mathrm{~s}$ & 2.90 & 4.65 & 6.19 \\
\hline & $2010 \mathrm{~s}$ & 2.10 & 4.57 & 2.89 \\
\hline \multirow{3}{*}{$R$} & $1960 \mathrm{~s}$ & 40.91 & 41.39 & 41.08 \\
\hline & $1980 \mathrm{~s}$ & 42.76 & 45.72 & 44.39 \\
\hline & $2010 \mathrm{~s}$ & 46.14 & 42.37 & 40.68 \\
\hline \multirow{3}{*}{$D$} & $1960 \mathrm{~s}$ & 1.45 & 1.67 & 1.69 \\
\hline & $1980 \mathrm{~s}$ & 1.43 & 1.69 & 1.61 \\
\hline & $2010 \mathrm{~s}$ & 1.38 & 1.66 & 1.61 \\
\hline
\end{tabular}

From the two paragraphs above, both quantity and structure of the streams were deeply degraded by urbanization, correspondingly, the spatial complexity of river network was also weakened. $D$ in highly urbanized area was the lowest, averaging 1.42 in the three periods, while the values were 1.67 and 1.64 in moderately and lowly urbanized areas. During the $1960 \mathrm{~s}-2010 \mathrm{~s}$, highly urbanized area shared the largest decreasing rate $(4.91 \%)$, followed by $4.73 \%$ and $0.42 \%$ in low and medium degrees of urbanization, respectively. Therefore, the pattern of river network weakened from spatially complex to simple, as urban areas expanded, and this tendency was dominant in highly urbanized area, where human activity was intensified.

\subsection{River evolution influenced by urbanization}

Natural river system has its own mechanism of evolution without disturbance of human activities, however, urbanization destroyed the stream in the form of invading and burying headwaters. Great disappearing tributaries caused impaired regulating and storing ability of the watershed, thus bringing about higher occurrence of flood disasters. To address this issue, human widened and dredged main streams to increase the discharging ability downstream. Therefore, this feedback control was found between river system and human activities, and stream structure changed with urbanization and restricted it in return, reaching final equilibrium through adjustment processes. This view is consistent with Chin's view that stream morphology would adjust to changed hydrologic conditions induced by urbanization and reach new stability regimes (2006). The river networks have been weakened a lot after new equilibrium. However, this equilibrium in this study was reached in view of the flood prevention of watershed, and the weakening pattern of river network will make potential fatal 
impact on the aquatic ecosystem, such as biological diversity and water quality in rivers, which are often ignored by the government. Once the river systems are degraded over threshold, the ability of auto-adjustment of river system is destroyed, thus causing catastrophic results.

In the past 50 years, quantity, structure and spatial complexity of river network in the YRD all exhibited downward tendency, and some indices would even have intensification of decreasing trend, such as water surface ratio. Therefore, the relationship between river system and human has not reached equilibrium, and more weakening river network in the YRD are expected as with urbanization development.

\section{Conclusions}

In this study, variations of stream structure in the past 50 years in the Yangtze River Delta were investigated, and evolution mechanisms of streams in the background of urbanization were discussed through analyzing stream characteristics in areas with different levels of urbanization. Some conclusions are given below.

(1) Tributaries contribute a majority to river system, but headwater has disappeared by a great deal in Hangjiahu intervened by fierce human activities, facing serious problems in stability of river structure.

(2) Quantitatively, drainage density $(D d)$ in the YRD showed overall downward tendency, with regions Wuchengxiyu, Hangjiahu and Yindongnan significantly decreasing by $20 \%$, and decreasing trend of water surface ratio (WSR) has intensified. Structurally, average width of main stream $(R)$ in Qinhuai River basin dramatically increased from 44.1 in the 1960 s to 73.2 in the 2010 s, and development coefficient $(K)$ of tributaries in Hangjiahu has decreased most, by $46.8 \%$ in the past 50 years. Besides, there exhibited a declining tendency in morphological complexity $(D)$ in the whole study area.

(3) The impact of urbanization on river network involves two aspects. On the one hand, urbanization affected the spatial pattern of stream. Highly urbanized area had minimum values of $D d, W S R, K$ and $D$, and highest $R$, indicating that human activities during urbanization weakened the river system by burying the headwater resulted declining spatial complexity. On the other hand, urbanization altered the process of river evolution. In highly urbanized areas, the main streams were preferentially protected, widened and dredged for alleviating flood risk, and main streams have been becoming dominant of river network. But in areas of medium level of urbanization, agricultural activities during the 1960s-1980s temporarily had increased the drainage density. In lowly urbanized areas, as the process of urbanization speeded up after the $1980 \mathrm{~s}$, headwaters were buried on a great deal, resulting in a sharp decrease of $D d$ and $K$ during the $1980 \mathrm{~s}-2010$ s.

\section{References}

Chen Dechao, Li Xiangping, Yang Jishan et al., 2002. Development of water system in the progress of urbanization of Shanghai and its influences to the city drainage. Urban Problems, (5): 31-35. (in Chinese)

Chin A, 2006. Urban transformation of river landscapes in a global context. Geomorphology, 79(3/4): $460-487$.

Dunne T, Leopold L B, 1978. Water in Environmental Planning. San Francisco: W.H.Freeman.

Elmore A J, Kaushal S S, 2008. Disappearing headwaters: Patterns of stream burial due to urbanization. Frontiers in Ecology and the Environment, 6(6): 308-312. 
Graf W L, 1977. Network characteristics in suburbanizing streams. Water Resources Research, 13(2), 459-463.

Gregory K J, Davis R J, Downs P W, 1992. Identification of river channel change due to urbanization. Applied Geography, 12(4): 299-318.

Hammer T. R., 1972. Stream channel enlargement due to urbanization. Water Resources Research, 8(6), 1530-1540.

Han Changlai, Mao Rui, 1997. The structure characteristics and the functional variation of the river systems in Taihu Lake catchment. Journal of Lake Science, 9(4): 300-306. (in Chinese)

Han Longfei, Xu Youpeng, Shao Yulong et al., 2013. Effect of urbanization on the stream structure and connectivity: A case study in the midlower reaches of the Qinhuai River. Journal of Lake Sciences, 25(3): 335-341. (in Chinese)

Han Longfei, Xu Youpeng, Yang Liu et al., 2015. Temporal and spatial change of stream structure in Yangtze River Delta and its driving forces during 1960s-2010s. Acta Geographica Sinica, 70(5): 819-827.

Horton Robert E, 1945. Erosional development of streams and their drainage basins: Hydrophysical approach to quantitative morphology. Geological Society of America Bulletin, 56(3): 275-370.

Huang Yilong, Wang Yanglin, Liu Zhenhuan et al., 2008. Stream construction characteristics in rapid urbanization area: Shenzhen city as a case. Geographical Research, 27(5): 1212-1220. (in Chinese)

Ji Xiaomin, Xu Youpeng, Han Longfei et al., 2014. Impacts of urbanization on river system structure: A case study on Qinhuai River Basin, Yangtze River Delta. Water Science \& Technology, 70(4): 671-677.

Li Yuanyuan, Li Jianqiang, Li Zongli et al., 2011. Issues and challenges for the study of the interconnected river system network. Resources Science, 33(3): 386-391. (in Chinese)

Marsh George Perkins, 1865. Man and Nature, or Physical Geography as Modified by Human Action. University of Washington Press.

Meyer J L, Wallace J B, Press M C et al., 2001. Lost linkages and lotic ecology: Rediscovering small streams. In: Ecology: Achievement and Challenge. The 41st Symposium of the British Ecological Society sponsored by the Ecological Society of America held at Orlando, Florida, USA, 10-13 April 2000. Blackwell Science, $295-317$.

Ni Jinren, Liu Yuanyuan, 2006. Ecological rehabilitation of damaged river system. Science and Technology Review, 24(7): 17-20. (in Chinese)

Strahler Arthur N, 1957. Quantitative analysis of watershed geomorphology. Eos, Transactions American Geophysical Union, 38(6): 913-920.

Tian Guangjin, Jiang Jing, Yang Zhifeng et al., 2011. The urban growth, size distribution and spatio-temporal dynamic pattern of the Yangtze River Delta megalopolitan region, China. Ecological Modelling, 222(3): $865-878$.

Vanacker Veerle, Molina Armando, Govers Gerard et al., 2005. River channel response to short-term human-induced change in landscape connectivity in Andean ecosystems. Geomorphology, 72(1): 340-353.

Xiao P F, Wang X H, Feng X Z et al., 2014. Detecting China's urban expansion over the past three decades using nighttime light data. IEEE Journal of Selected Topics in Applied Earth Observations and Remote Sensing, 7(10): 4095-4106.

$\mathrm{Xu}$ Guanglai, $\mathrm{Xu}$ Youpeng, Wang Liuyan, 2013. Temporal and spatial changes of river systems in Hangzhou-Jiaxing-Huzhou Plain during 1960s-2000s. Acta Geographica Sinica, 68(7): 966-974. (in Chinese)

$\mathrm{Xu}$ Youpeng, 2012. Impacts of Urbanization of the Yangtze River Delta Region on River System in Basins and Hydrological Processes. Beijing: Science Press. (in Chinese)

Yang Kai, Yuan Wen, Zhao Jun et al., 2004. Stream structure characteristics and its urbanization responses to tidal river system. Acta Geographica Sinica, 59(4): 557-564. (in Chinese)

Yuan Wen, Yang Kai, Tang Min et al., 2005. Stream structure characteristics and their impact on storage and flood control capacity in the urbanized plain river network. Geographical Research, 24(5): 717-724. (in Chinese)

Yuan Wen, Yang Kai, Wu Jianping, 2007. River structure characteristics and classification system in river network plain during the course of urbanization. Scientia Geographica Sinica, 27(3): 401-407. (in Chinese)

Zhang Shixia, Guo Yakun, Wang Ziwen, 2015. Correlation between flood frequency and geomorphologic complexity of rivers network: A case study of Hangzhou China. Journal of Hydrology, 527: 113-118.

Zhou Hongjian, Shi Peijun, Wang Jingai et al., 2008. River network change and its ecological effects in Shenzhen region in recent 30 years. Acta Geographica Sinica, 63(9): 969-980. (in Chinese)

Zhou Hongjian, Wang Jingai, Yue Yaojie et al., 2006. Assessment of flood hazard based on river network change: Taking the Beijing-Tianjin segment of Yongding River watershed as an example. Journal of Natural Disasters, 15(6): 45-49. (in Chinese) 